\title{
Highlights from a model for remote delivery of pharmacy laboratory courses: Design, implementation and student feedback
}

\author{
D. Hammoudi Halat*, M. Cherfan, N. Mourad, M. Rahal
}

School of Pharmacy, Lebanese International University, Lebanon

\author{
Keywords \\ Remote Learning \\ Laboratory Courses \\ Pharmaceutical Sciences \\ Assessment \\ Lebanon \\ *Corresponding author: \\ dalal.hammoudi@liu.edu.lb
}

\begin{abstract}
Summary: Laboratory courses constituted a major challenge to remote learning, particularly with limited previous experience in virtual delivery. This case study aims to describe a model used for remote delivery of laboratory courses for students at Lebanese International University, School of Pharmacy, and to report student experiences, perceptions and attitudes through a structured questionnaire. Google Classroom was used as the learning platform, with synchronous and asynchronous teaching. Videos were used to simulate experiments; assignments, reports and quizzes were used for assessments. A total of 329 students responded to the questionnaire. The majority reported a good experience and satisfaction; $62 \%$ believed that simulation videos were of good quality, easy to access and were of reasonable length. Gaps detected were deficient practice (44\%), lack of experience with instruments (46\%), and poor motor skills (49\%). Students expressed preference for experiments videotaped by their instructors. Overall, this model was well received, and provided an alternative to remotely deliver practical courses. This study assists in preparation of future remote laboratory learning activities.
\end{abstract}

\section{Background and Context}

The pandemic caused by the coronavirus disease 2019 (COVID-19) has had serious implications on pharmacy academia, disrupting fundamental teaching elements that are long established and commonly implemented (Brazeau, 2020). Around the globe, pharmacy education was suddenly shifted towards remote solutions like virtual learning, videoconferencing, social media and telecommunication to tackle the prolonged interruption to physical classes (Dedeilia et al., 2020). Laboratory courses constituted a specific challenge to remote instruction, and the need to achieve learning outcomes from the practical courses via a fully virtual experience is difficult. Moreover, the finding of adequate remote alternatives to the hands-on experience that students gain from practical laboratory work is problematic (Hallal, HajjHussein, \& Tlais, 2020).
At the School of Pharmacy, Lebanese International University, three pharmaceutical sciences laboratory courses are offered during the spring term, they are: quantitative analysis, pharmaceutical analysis and biotechnology applications, and compounding laboratory. When COVID-19 struck in Lebanon, a government lockdown was quickly implemented on 12th March 2020; students had physically experienced less than three weeks of the total ten-week timeline of these courses. The department, faculty, and laboratory assistants aimed to seek a suitable strategy for the completion of laboratory courses through remote teaching.

This case study aims to summarise the model used at the School of Pharmacy, Lebanese International University, for remote delivery of pharmacy laboratory courses. The case study also describes the outcomes of an assessment 
questionnaire intended to get students' feedback regarding these courses. The evaluation of students' experience, perceptions and attitudes towards remote laboratory learning is crucial for strategic planning and preparedness for any remote laboratory learning activities in the future.

\section{Educational Description}

Following guidance from the University regarding online learning, the School of Pharmacy carried out a prompt transition plan to remote education soon after lockdown started. In this plan, Google Classroom was chosen as the learning platform for communication with students. Both synchronous (live meetings) and asynchronous (recorded lectures) teaching methods were applied. These changes, however, had to be further customised to accommodate laboratory courses, each of which had a theoretical component and an applied (practical) one. During laboratory courses students (either individually or in groups of two to three) usually realise the actual bench experiments, record results, submit written reports, and sit for practical/written exams to be assessed. The modified plan for these courses consisted of the following elements:

1. Theoretical components were recorded as voice-over PowerPoint presentations, shared with students, and discussed during live meetings, scheduled at the same time slot as when the original laboratory course was offered;

2. Practical components were substituted by YouTube videos shared with students to simulate experiments;

3. Laboratory reports were requested based on a repertoire of experimental results previously collected by instructors and provided to students;

4. Formative assessments included reports and assignments. Summative assessments included quizzes and final exams administered via Google Classroom, as well as an exam involving questions about a videotaped experiment, to replace the actual practical exam normally held in the laboratory to test practical skills.

To assess students' experience with adopted methods of remote delivery of laboratory courses, a questionnaire evaluating attitudes and perceptions was created. A literature review was undertaken to identify existing potential data based on previous studies evaluating students' experiences with such remote courses (Dunham, Ghirtis, \& Beleh, 2012; Park \& Shrewsbury, 2016). The survey was anonymous and administered electronically online, using Google Forms, towards the end of laboratory sessions. The survey was piloted, whereby laboratory instructors and ten students reviewed it for clarity, and changes were made to remove redundant questions and optimise the tool. The final questionnaire consisted of eight sections with a total of 57 questions. First, students' background information was collected; then, feedback on the online learning was evaluated using a Likert scale of 1-5, ( 1 = strongly disagree and $5=$ strongly agree). The different sections addressed students' laboratory experiences, perceptions towards simulation videos, development of skills, meeting intended learning outcomes, assessments, satisfaction and attitudes towards remote learning in laboratory courses. An openended question was added at the conclusion of the questionnaire to capture students' additional comments. The study protocol was approved by the Research Ethics Committee of the School. Categorical data based on Likert scale were analysed descriptively using frequency and percentage. Students' feedback, which described their views and experiences of online learning were included. The comments received served as a reference to improve the content, design and user satisfaction towards remote laboratory learning. A total of 503 students registered in laboratory courses across eight campuses during the spring term, in second- or third- year of the Bachelor of Pharmacy programme, were invited to complete the questionnaire.

\section{Outcomes and Recommendations}

A structured, consistent format is key for satisfying laboratory courses' outcomes. With no standardised approach to realise remote instruction of laboratory courses in pharmacy schools, accessible, functional, and useful methods during COVID-19 are compulsory, especially where practical pharmaceutical science courses constitute an integral part of the curriculum.

A total of 329 students responded to the questionnaire, $63 \%$ were in second-year, and $83 \%$ had not previously taken remote courses. The majority had average computer skills and accessed remote courses from laptops (74\%) and/or smart phones (85\%). Regarding simulation videos, $62 \%$ of students believed these had good quality, were easy to access, and were of reasonable length, while $70 \%$ agreed there was clarity in the delivery. Half of the students believed remote delivery met experimental goals, and were able to write structured reports; also, $47 \%$ were able to analyse experimental results. Only $20.1 \%$ disagreed and considered themselves unable to correctly perform laboratory calculations. Students were generally positive about the assessments, with $66 \%$ describing them as clear, $59 \%$ as efficient, and $56 \%$ as providing timely and effective feedback. Students showed satisfaction with clear deadlines (77\%), flexibility (65\%), and motivation of self-directed learning (52\%). Nevertheless, gaps detected were deficient practice $(44 \%)$, lack of experience with devices $(46 \%)$, and poor learning of fine motor skills (49\%). Compared to conventional courses, only $26.8 \%$ of students agreed about learning practical skills like mixing and compounding, while $20.4 \%$ agreed about learning motor skills. Furthermore, $49 \%$ of students preferred at least one on-campus practical exam. 
With students' preference to have experiments videotaped by instructors, the School plans to implement this strategy for future terms.

This model provides an alternative to remotely deliver practical courses, which was, overall, well-received by students. While schools continue to create contingency plans to accommodate the effects of COVID-19, and while remote instruction abridges the ultimate spirit of practical courses, it may still be helpful to address student needs with in-person laboratorybased practicals.

\section{References}

Brazeau, G.A. (2020). Lessons Learned and Brighter Opportunities for Pharmacy Education Amid COVID-19. American Journal of Pharmaceutical Education, 84(6), https://doi.org/10.5688/ajpe8230

Dedeilia, A., Sotiropoulos, M.G., Hanrahan, J.G., Janga, D. Dedeilias, P., \& Sideris, M. (2020) Medical and Surgical Education Challenges and Innovations in the COVID-19 Era: A Systematic Review. In Vivo, 34, 1603-1611, https://doi.org/10.21873/invivo.11950

Dunham, M.W., Ghirtis, K., \& Beleh, M. (2012) The use of virtual laboratories and other web-based tools in a drug assay course. American Journal of Pharmaceutical Education, 76(5) 84, https:// doi.org/10.5688/ajpe76584

Hallal, K., HajjHussein, H., \& Tlais, S. (2020) A Quick Shift from Classroom to Google Classroom: SWOT Analysis. Journal of Chemical Education, https://doi.org/10.1021/acs.jchemed.0c00624

Park, H.L., \& Shrewsbury, R.P. (2016) Student Evaluation of Online Pharmaceutical Compounding Videos. American Journal of Pharmaceutical Education, 80(2) 30, https://doi.org/10.5688/ ajpe80230 